\title{
Indicadores de calidad en atención a fractura de cadera tras la implementación de un equipo de ortogeriatría
}

\author{
Quality indicators in hip fracture care after the implementation of an orthogeriatrics team
}

\author{
Viveros-García JC, * Guillermo-Nuncio EA, ${ }^{\ddagger}$ Nieto-Sandoval HR, ${ }^{\S}$ Baldenebro-Lugo LS \\ Hospital Regional ISSSTE, León, México.
}

RESUMEN. Introducción: La incidencia de fractura de cadera se incrementará radicalmente en las siguientes décadas. Se ha asociado a una mortalidad y morbilidad elevada. Los programas de ortogeriatría en hospitales públicos han demostrado mejorar los resultados asistenciales. Hay poca evidencia en México sobre el impacto de la ortogeriatría y el apego a indicadores de calidad. Objetivo: Comparar el apego a indicadores de calidad en fractura de cadera antes y después de la implementación de un equipo de ortogeriatría comparado con el modelo tradicional. Material y métodos: Estudio cuasiexperimental realizado en León, México. Se comparó la adherencia a indicadores de calidad antes y después de un equipo interconsultante de ortogeriatría. Los indicadores medidos fueron: demora quirúrgica, tratamiento para osteoporosis, movilización temprana, tipo de implante y delirium. Resultados: Incluimos 83 pacientes. La demora quirúrgica se disminuyó de 144 a 116 horas (28 horas), se incrementó el porcentaje de pacientes con cirugía temprana de 7.9 a $18 \%$, además se incrementó el porcentaje de pacientes con tratamiento para osteoporosis y se logró disminuir la incidencia de delirium. Conclusión: El apego a indicadores es bajo; sin embargo, se logró incrementar discretamente el
ABSTRACT. Introduction: The incidence of hip fracture will increase dramatically in the coming decades. It has been associated with high mortality and morbidity. Orthogeriatric programs have improved outcomes in fragility hip fracture worldwide. There is little evidence in Mexico on the impact of orthogeriatrics and adherence to quality indicators. Objective: To compare adherence to quality indicators in hip fracture before and after the implementation of an orthogeriatrics team compared to the traditional model. Material and methods: Quasiexperimental study conducted in León, Mexico. Adherence to quality indicators before and after an interconsultant orthogeriatrics team was compared. The indicators measured were: surgical delay, treatment for osteoporosis, early mobilization, type of implant and delirium. Results: We included 83 patients. The surgical delay was reduced from 144 to 116 hours (28 hours), the percentage of patients with early surgery was increased from 7.9 to $18 \%$, in addition the percentage of patients with treatment for osteoporosis was increased and the incidence of delirium was reduced. Conclusion: Adherence to indicators is low, however, it was possible to discreetly increase adherence

\section{Nivel de evidencia: IV}

* Geriatra, Equipo de Ortogeriatría.

* Residente de tercer año de Traumatología.

$\S$ Adscrito al Servicio de Traumatología.

" Geriatra. Jefatura de Investigación Clínica.

\section{Correspondencia:}

Juan Carlos Viveros-García

Hospital Regional ISSSTE, León, México.

Av. Pradera Núm. 1101, Col. Aztecas, León, C.P. 37520, Guanajuato, México.

E-mail: drviveros.geriatria@gmail.com

Recibido: 15-03-2020. Aceptado: 18-01-2021.

Citar como: Viveros-García JC, Guillermo-Nuncio EA, Nieto-Sandoval HR, Baldenebro-Lugo LS. Indicadores de calidad en atención a fractura de cadera tras la implementación de un equipo de ortogeriatría. Acta Ortop Mex. 2021; 35(2): 181-187. https://dx.doi.org/10.35366/101863

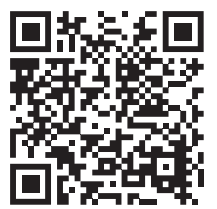


apego a estos indicadores. Se deben generar políticas internas en los hospitales públicos para mejorar esta adherencia y ver su impacto en los resultados a largo plazo.

Palabras clave: Fractura, cadera, osteoporosis, geriatría, atención.

\section{Introducción}

Las fracturas por fragilidad afectan a uno de cada cinco hombres y a una de cada tres mujeres mayores de 50 años. ${ }^{1}$ Dentro de éstas, la fractura de cadera (FC) es la más grave. ${ }^{2}$ Su prevalencia se ha incrementado en los últimos años ${ }^{3,4,5}$ y se estima que para el año 2050 habrá más de 6,000,000 de casos por año en todo el mundo. ${ }^{6}$ En la Ciudad de México la tasa anual FC es de 169 casos por cada 100,000 mujeres y de 98 por cada 100,000 hombres. ${ }^{7}$ Además de su frecuencia, otro de los factores que la vuelve catastrófica es su relación con el desarrollo de dependencia y de tener una mortalidad cercana a $25 \%$ en el primer año. ${ }^{8,9}$

La FC es la principal causa de muerte en adultos mayores hospitalizados en centros de trauma. ${ }^{10}$ De los sobrevivientes, sólo tres de cada cuatro recuperarán la movilidad que tenían previo a la fractura. ${ }^{11}$ Por otra parte, los costos en FC son muy altos. En México se han calculado los costos directos en la fase aguda de la fractura que se encuentran entre $\$ 2,000$ y $\$ 13,000$ dólares estadounidenses. ${ }^{7,12}$

En las últimas décadas sociedades académicas y científicas han publicado guías de práctica clínica y han recomendado indicadores de calidad (IC) que han demostrado mejorar los resultados asistenciales de la FC..$^{13,14,15}$ El modelo ortogeriátrico es uno de estos indicadores, , ${ }^{16,17,18}$ demostrando tener impacto positivo en la mortalidad, recuperación funcional y costos. ${ }^{18,19,20,21,22}$ Considerando que el paciente con FC es de alto riesgo de complicaciones, con una comorbilidad significativa que incluye la fragilidad y el deterioro cognitivo, el geriatra tiene las competencias para el manejo de estas entidades, por lo que cada vez es más frecuente su inclusión en los equipos multidisciplinarios. 11,23.24,25

Existe distintos modelos de atención ortogeriátrica, los más frecuentes son el comanejo o manejo conjunto y los equipos interconsultantes. ${ }^{26}$ En América Latina existen pocos servicios de este tipo y la evidencia de la región es muy escasa. ${ }^{27}$ Nuestro objetivo fue evaluar el apego a indicadores de calidad en el paciente con fractura de cadera por fragilidad tras la implementación de un equipo de ortogeriatría en un hospital del sistema público de salud, en comparación con el manejo tradicional de traumatología y ortopedia. Los objetivos secundarios fueron determinar las características sociodemográficas de los pacientes con FC en nuestro hospital, además de identificar las complicaciones más importantes, mortalidad y estancia hospitalaria. to these indicators. Internal policies must be generated in public hospitals to improve this adherence and see its impact on long-term outcomes.

Keywords: Fracture, hip, osteoporosis, geriatrics, attention.

\section{Material y métodos}

Este estudio se llevó a cabo en un centro de referencia de tercer nivel para trabajadores del gobierno. Se atienden en promedio 50 fracturas de cadera por fragilidad por año. Se realizó un estudio cuasiexperimental comparando el apego a los indicadores de calidad en el modelo tradicional de traumatología (fase retrospectiva) contra el modelo de interconsulta de ortogeriatría (prospectivo) (Figura 1).

Incluimos pacientes de 60 años o más con diagnóstico de fractura de cadera por fragilidad, definida como aquélla que es consecuencia de un traumatismo de baja energía. Se excluyeron pacientes con fracturas en terreno patológico por neoplasias o enfermedad metastásica. Se eliminaron los casos en los que en la fase retrospectiva tenían expedientes con datos incompletos, así como los que solicitaron alta voluntaria en la fase prospectiva.

En el modelo de atención tradicional los pacientes con FC ingresaban a cargo exclusivo del cirujano ortopédico, quien estaba a cargo del paciente durante toda la hospitalización. Se solicitaba valoración preoperatoria al servicio de medicina interna una vez que se ingresaba a piso y posteriormente en caso de que el clínico a cargo lo considerara necesario. A partir de Abril de 2018 se inició con un programa de interconsulta de geriatría. Este modelo incluyó a un geriatra en el turno matutino de Lunes a Viernes. Geriatría estuvo a cargo de la valoración preoperatoria desde el servicio de urgencias así como del manejo médico de las comorbilidades, vigilancia de las mismas en el postquirúgico y manejo de las complicaciones no quirúrgicas. El traumatólogo se encargó de la programación quirúrgica, elección del implante a utilizar así como del inicio de movilización fuera de cama, del inicio de carga y rehabilitación.

Se midieron en ambas fases del estudio las variables sociodemográficas, comorbilidad, características de la marcha usando la Categoría Funcional Ambulatoria (Functional Ambulatory Classification o FAC), ${ }^{28}$ tratamiento para osteoporosis previo a la fractura, funcionalidad y estado cognitivo al ingreso.

Durante la fase aguda se midió la adherencia a seis indicadores de calidad sugeridos por la guía de práctica clínica

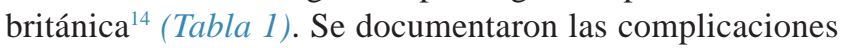
intrahospitalarias, la mortalidad y la estancia hospitalaria. Este protocolo recibió autorización por el comité local de ética e investigación con el número de registro CEI2619.

Análisis estadístico: muestra no probabilística de casos consecutivos. Se usó la paquetería SPPS versión 24. Las 


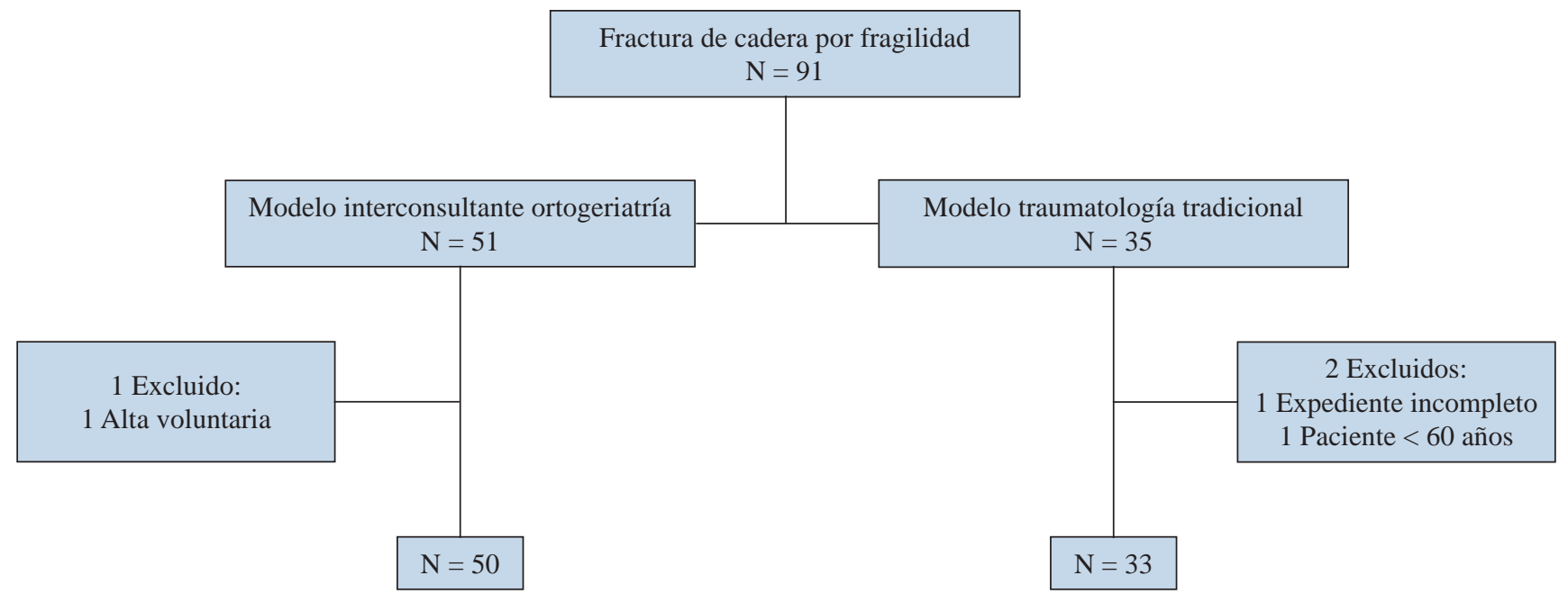

Figura 1: Población del estudio.

Tabla 1: Indicadores de calidad en fractura

de cadera recomendados por la guías National

Institute for Health and Care Excellence.

Evaluación geriátrica

Cirugía en menos de 48 horas

Rehabilitación y movilización temprana

Tratamiento para osteoporosis al alta

Escrutinio y prevención del delirium

Cirugía correcta para el tipo de fractura

a) Intracapsulares tratadas con prótesis total o parcial

b) Transtrocantérica tratada con clavocentromedular o placa deslizante de cadera

c) Subtrocantérica tratada con clavocentromedular

NICE = National Institute for Health and Care Excellence.

variables continuas se reportan en medias \pm desviaciones estándar o medianas con rango intercuartil según corresponda. Los indicadores de calidad se compararon a través de pruebas no paramétricas. Se consideraron resultados estadísticamente significativos aquéllos con un valor de $\mathrm{p}$ igual o menor de 0.05 .

\section{Resultados}

Entre Abril de 2017 y Marzo de 2019 se ingresó a nuestro hospital un total de 91 fracturas de cadera, se excluyeron tres pacientes del análisis final, dejando un total de 88 pacientes, 38 tratados con el modelo tradicional y 50 con un equipo interconsultante de ortogeriatría

Las características basales de los grupos se muestran en la Tabla 2. Tres de cada cuatro pacientes fueron catalogados con un riesgo perioperatorio alto. La comorbilidad fue similar en ambos grupos, exceptuando la fragilidad y el deterioro cognitivo que se reportaron con más frecuencia en el grupo ortogeriátrico (Tabla 3). Los resultados asistenciales se muestran en la Tabla 4, la mayoría de las complicaciones fueron menores en el grupo ortogeriátrico sin lograr signifi-

\begin{tabular}{|c|c|}
\hline \multicolumn{2}{|c|}{$\begin{array}{l}\text { Tabla 2: Características demográficas y funcionalidad } \\
\text { prefractura, tipo de fracturas e historia previa } \\
\text { de fracturas por fragilidad. } \mathbf{N}=\mathbf{8 8} \text {. }\end{array}$} \\
\hline & n (\%) \\
\hline Edad & $77.4 \pm 9.67$ \\
\hline Femenino & $62(70.5)$ \\
\hline $\begin{array}{l}\text { Marcha independiente previo a la fractura (FAC* } \\
\text { tres o superior) }\end{array}$ & $46(52.2)$ \\
\hline Tratamiento para osteoporosis previo a la fractura & $9(10.2)$ \\
\hline Funcionalidad previo a la fractura ${ }^{\ddagger}$ & $90(60-95)$ \\
\hline Historial de fracturas por fragilidad & $27(30.7)$ \\
\hline \multicolumn{2}{|l|}{ Tipo de fractura } \\
\hline Intracapsular no desplazada & $16(18.2)$ \\
\hline Intracapsular desplazada & $12(13.6)$ \\
\hline Transtrocantérica & $51(58.0)$ \\
\hline Subtrocantérica & $9(10.2)$ \\
\hline \multicolumn{2}{|c|}{$\begin{array}{l}\text { * Functional Ambulatory Classification (FAC). FAC } 0=\text { no camina. FAC } 1 \\
\text { = requiere asistencia continua de otra persona para soportar el peso. FAC } 2= \\
\text { requiere contacto intermitente ligero para mantener el balance. FAC } 3=\text { Puede } \\
\text { deambular en superficies lisas sin contacto o apoyo. FAC } 4=\text { puede deambular } \\
\text { en superficies lisas, pero requiere ayuda en escaleras. FAC } 5=\text { deambulación } \\
\text { independiente en todas las superficies incluyendo escaleras. } \\
\text { ₹ Índice de Barthel; mediana (rango intercuartil). }\end{array}$} \\
\hline
\end{tabular}

cancia estadística. También hubo una menor estancia hospitalaria. Con respecto a los indicadores de calidad, la media de demora quirúrgica fue 28 horas menor en el grupo ortogeriátrico, además hubo mayor porcentaje de pacientes que se operaron en las primeras 48 horas de estancia hospitalaria. Encontramos diferencia estadísticamente significativa en el número de pacientes que se sentaron al día siguiente de la cirugía y los que recibieron tratamiento para osteoporosis al egreso.

Los implantes que se utilizaron con más frecuencia fueron la hemiprótesis en las fracturas intracapsulares y la placa deslizante de cadera (DHS) en las transtrocantéricas y clavo centromedular en las subtrocantéreas (Figura 2). 


\section{Discusión}

Los programas de ortogeriatría en México son modelos relativamente nuevos; sin embargo, sus beneficios se empiezan a difundir y se han implementado nuevas unidades en los últimos años. ${ }^{29}$ En otros países de América Latina ya hay unidades bien establecidas y han demostrado tener un impacto positivo en los resultados asistenciales en salud. ${ }^{30,31,32}$

El modelo de un servicio interconsultante de ortogeriatría es el menos efectivo de los modelos; sin embargo, también suele ser el primer paso en el desarrollo de un servicio de manejo conjunto debido a la complejidad de un servicio de estas características. ${ }^{26}$ Este artículo corresponde a los resultados de la primera fase en el desarrollo de un servicio de ortogeriatría.

Encontramos que los pacientes con FC en nuestro hospital son un poco más jóvenes que los pacientes de economías desarrolladas y sistemas de salud más eficientes, esto como resultado de una transición demográfica en México un poco más tardía. ${ }^{33,34,35,36,37}$ Chile, Colombia y Ecuador tienen una edad similar a la observada en nuestro estudio. ${ }^{31,38,39}$ Otro hallazgo importante en nuestro estudio fue que la fragilidad y el deterioro cognitivo se reportaron con más frecuencia en el grupo de ortogeriatría, esto lo atribuimos a que la valoración geriátrica integral incluye escrutino intencionado de estos dos síndromes, mientras que no suele ser considerado en la historia clínica ortopédica. Este fenómeno de mayor detección de síndromes geriátricos en modelos de ortogeriatría es consistente con el reporte de Baroni y colaboradores, donde además se logró disminuir el número de interconsultas a otros servicios. ${ }^{40}$

Con respecto a los indicadores de calidad, todos los pacientes en el modelo ortogeriátrico fueron valorados por el geriatra del turno matutino. El segundo indicador de calidad evaluado fue la demora quirúrgica, que se logró disminuir 28 horas (144 vs. 116 horas). Además se incrementó el porcentaje de pacientes operados en las primeras 48 horas a 18\%; sin embargo, este indicador aún está muy por debajo de lo publicado en países desarrollados. ${ }^{9}$ Existe poca evidencia de la demora quirúrgica en países latinoamericanos, pero reportes en Chile publicaron una media de 19 días. $^{31}$

Tabla 3: Demografía y comorbilidades en los pacientes con fractura de cadera por fragilidad en ambos grupos.

\begin{tabular}{|c|c|c|c|}
\hline & $\begin{array}{l}\text { Modelo de interconsulta ortogeriátrico } \\
\qquad \mathrm{N}=50, \mathrm{n}(\%)\end{array}$ & $\begin{array}{l}\text { Modelo de traumatología tradicional } \\
\qquad \mathrm{N}=38, \mathrm{n}(\%)\end{array}$ & $\mathrm{p}$ \\
\hline Edad & $77 \pm 9.66$ & $82.11 \pm 9.12$ & $0.020 *$ \\
\hline Género femenino & $35(70)$ & $27(71.1)$ & $0.553^{\ddagger}$ \\
\hline Riesgo quirúrgico alto (ASA III o IV) & $36(72)$ & $29(76.3)$ & $0.473^{\S}$ \\
\hline Tratamiento previo para osteoporosis & $7(14)$ & $2(5.3)$ & $0.163^{\S}$ \\
\hline Funcionalidad (Indice Barthel) & $90(60-95)$ & NR & - \\
\hline Historia de fracturas por fragilidad & $20(40)$ & $7(18.4)$ & $0.025^{\ddagger}$ \\
\hline Diabetes mellitus & $22(44)$ & $15(39.5)$ & $0.418^{\ddagger}$ \\
\hline Hipertensión arterial sistémica & $32(64)$ & $21(55.3)$ & $0.271^{\ddagger}$ \\
\hline Enfermedad renal crónica estadio 3-5 & $12(24)$ & $5(13.2)$ & $0.158^{\ddagger}$ \\
\hline Cáncer & $3(6)$ & $14(36.8)$ & $0.010^{\ddagger}$ \\
\hline Enfermedades reumatológicas & $3(6)$ & $6(15.8)$ & $0.126^{\S}$ \\
\hline Deterioro cognitivo & $13(26)$ & $2(5.3)$ & $0.009^{\S}$ \\
\hline Fragilidad & $31(62)$ & $13(34.2)$ & $0.009^{\ddagger}$ \\
\hline
\end{tabular}

\begin{tabular}{|c|c|c|c|}
\hline & $\begin{array}{l}\text { Modelo de interconsulta ortogeriátrico } \\
\qquad \mathrm{N}=50, \mathrm{n}(\%)\end{array}$ & $\begin{array}{l}\text { Modelo de traumatología tradicional } \\
\qquad \mathrm{N}=38, \mathrm{n}(\%)\end{array}$ & $\mathrm{p}$ \\
\hline Complicaciones mayores & $34(68)$ & $26(68.4)$ & $0.576^{*}$ \\
\hline Delirium & $20(40)$ & $18(47.4)$ & $0.318^{\ddagger}$ \\
\hline Úlceras por presión & $17(34)$ & $7(18.4)$ & $0.082^{\ddagger}$ \\
\hline Neumonía & $5(10)$ & $4(10.5)$ & $0.602^{\pi}$ \\
\hline Anemia & $24(48)$ & $18(47.4)$ & $0.562^{\ddagger}$ \\
\hline Infección de vías urinarias & $10(20)$ & $4(10.5)$ & $0.182^{\ddagger}$ \\
\hline Mortalidad hospitalaria & $3(6)$ & $1(2.6)$ & $0.161^{\S}$ \\
\hline Estancia hospitalaria (días) & $7(5-9)$ & $8.46(5.5-9.5)$ & 0.463 \\
\hline
\end{tabular}


A. Intracapsulares

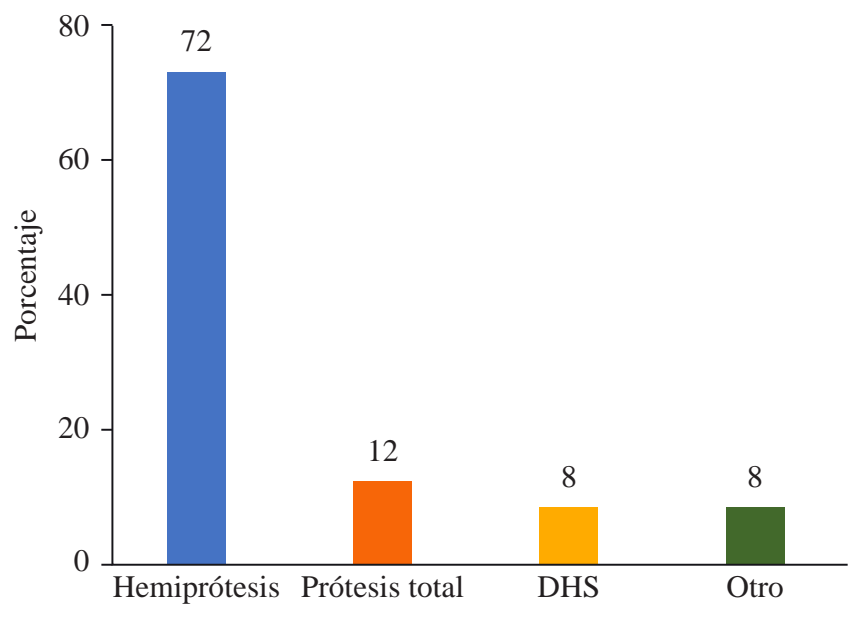

B. Transtrocantéreas

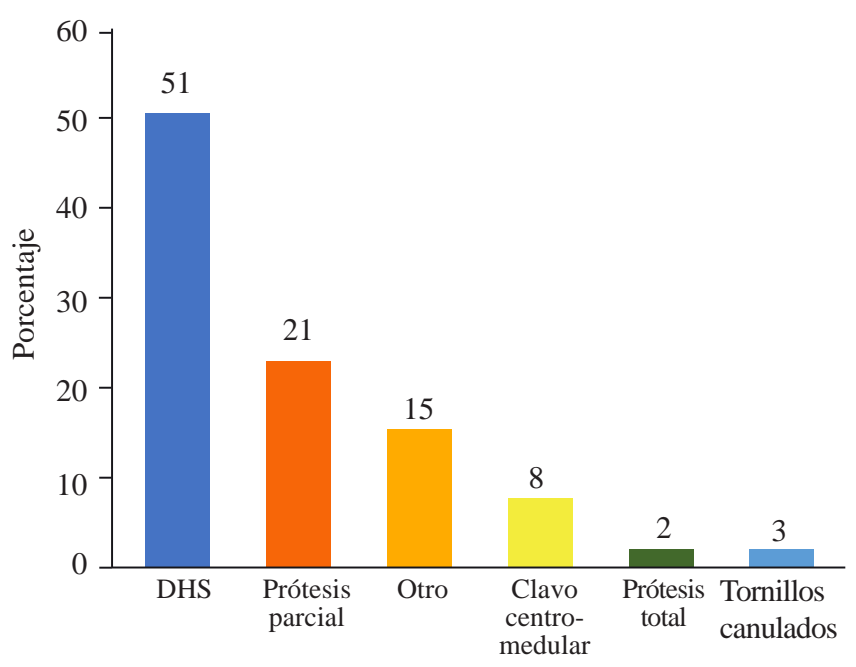

C. Subtrocantéreas

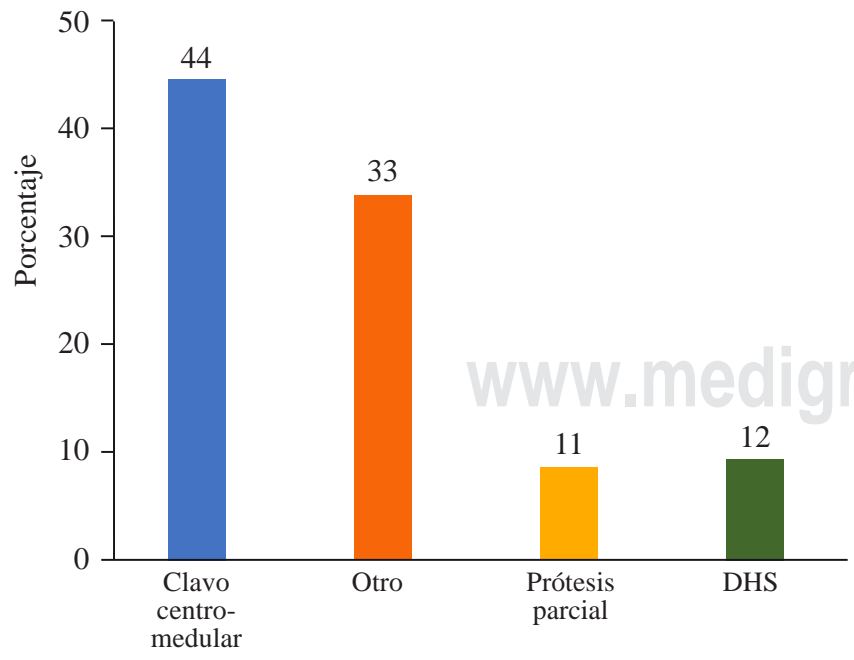

Figura 2: Implantes utilizados por tipo de fractura.

Otro = placa angulada u otros menos frecuentes .

DHS = Dynamic hip screw o placa deslizante de cadera .
El tercer indicador fue la movilización, sedentación así como el inicio de carga parcial a tolerancia. En nuestro hospital los pacientes no inician bidedestación durante la hospitalización de manera regular; sin embargo, sí se logró iniciar sedentación en 52\% de los casos. En otros países como España o Inglaterra, entre 64 y $80 \%$ de los pacientes se movilizan fuera de cama al día siguiente de la cirugía ${ }^{33,34}$ y tres de cada cuatro ya han iniciado deambulación para el momento en que son egresados. ${ }^{11}$ Esta brecha con respecto al inicio de carga se debe al modelo específico de tratamiento adoptado por nuestro hospital, hipotéticamente, para evitar complicaciones postquirúrgicas en los implantes utilizados, aunque existe poca evidencia científica en este punto, ya se está trabajando para cambiar este modelo hacia la movilización temprana.

El cuarto indicador de calidad medido fue el tratamiento para osteoporosis al egreso, que se incrementó de 0 a $40 \%$. Esta intervención es de suma importancia si consideramos que el riesgo de refractura es mucho mayor en las primeras semanas posteriores al egreso. ${ }^{41,42}$ Por último, los implantes utilizados con base en el tipo de fractura tienen una adherencia baja debido a cuestiones administrativas, ya que nuestro hospital está sujeto a disponibilidad de los dispositivos por parte del proveedor, además de licitaciones a nivel central. Particularmente el clavo centromedular no está licitado para el cuadro básico, por lo que su adquisición está sujeta a una compra extraordinaria, por ende, es un proceso burocrático complejo, lo que limita su uso en fracturas transtrocantéreas o subtrocantéreas.

La adherencia a estos indicadores se ha asociado a una mejoría en los resultados asistenciales, además de una menor tasa de complicaciones en el corto y largo plazo. Nuestro hospital tiene un porcentaje alto de complicaciones, particularmente delirium. Éste es el quinto indicador de calidad evaluado. El delirium se presentó en $47 \%$ en el modelo tradicional y en $40 \%$ en el modelo ortogeriátrico. Países desarrollados muestran tasas de 25 a 32\% de esta complicación. ${ }^{43,44}$

Los últimos dos puntos a discutir son las úlceras por presión y la mortalidad. Las úlceras se presentaron en $18 \%$ en el modelo tradicional vs. 34\% en el modelo ortogeriátrico. Esto se ha atribuido a una búsqueda sistemática de esta complicación como parte de la evaluación geriátrica integral; sin embargo, es una tasa muy alta y sumamente preocupante. Los registros nacionales de fractura de cadera en Europa reportan el desarrollo de úlceras por presión entre 3 y $6 \%{ }^{9,34}$ Consideramos que disminuir la demora quirúrgica y aumentar la movilización fuera de cama tendrá impacto en este indicador. Por último, la mortalidad intrahospitalaria fue similar a lo que se ha reportado en la literatura mundial; sin embargo, aún no tenemos datos sobre los resultados en el mediano y largo plazo.

La principal fortaleza del estudio es que, hasta donde los autores tienen conocimiento, es el primer trabajo mexicano en integrar la evaluación del paciente ortopédico por un geriatra e indicadores de calidad. Por otra parte, al ser la 


\begin{tabular}{|c|c|c|c|}
\hline & $\begin{array}{l}\text { Modelo de interconsulta ortogeriátrico } \\
\qquad \mathrm{N}=50, \mathrm{n}(\%)\end{array}$ & $\begin{array}{l}\text { Modelo de traumatología tradicional } \\
\qquad \mathrm{N}=38, \mathrm{n}(\%)\end{array}$ & $\mathrm{p}$ \\
\hline Demora quirúrgica (horas)* & $116(62.25-160.1)$ & $144(72-168)$ & $0.14^{\ddagger}$ \\
\hline Cirugía en menos de 48 horas & $9(18)$ & $3(7.9)$ & 0.227 \\
\hline Sedentación al día siguiente de la cirugía & $26(52)$ & $0(0)$ & $\mathrm{p}<0.01^{\pi}$ \\
\hline Carga parcial al tolerancia postquirúrgica & $0(0)$ & $0(0)$ & - \\
\hline Delirium & $20(40)$ & $18(47.4)$ & $0.318^{\|}$ \\
\hline Tratamiento para osteoporosis al alta & $20(40)$ & $0(0)$ & $\mathrm{p}<0.01^{9}$ \\
\hline \multicolumn{4}{|l|}{ Apego quirúrgico a guías NICE } \\
\hline Intracapsulares & $6(40)$ & $9(60)$ & $0.304^{\pi}$ \\
\hline Transtrocantérea & $22(59)$ & $11(55)$ & $0.618^{\natural}$ \\
\hline Subtrocantérea & $4(50)$ & $1(100)$ & $1.0^{4}$ \\
\hline
\end{tabular}

primera fase de un programa sirve de base para generar políticas de mejora y fomentar el desarrollo de programas de ortogeriatría primero que nada en nuestro sistema de salud y, de ser posible, se someterá a consideración en otros hospitales mexicanos. Las debilidades del estudio son el tamaño pequeño de la muestra, la baja estandarización terapéutica de estas fracturas, y la diferencia de pacientes incluidos en cada grupo. Es un trabajo retrospectivo y que no se compara con otros sistemas de salud.

\section{Conclusiones}

El apego a indicadores de calidad en nuestro hospital es bajo; sin embargo, se logró incrementar discretamente el apego a estos indicadores en disminución de demora quirúrgica, mejora de movilización temprana e inicio de tratamiento para la osteoporosis (Tabla 5).

Referencias

1. International Osteoporosis Foundation IO. La Carga Global de la Osteoporosis en Cifras. 2016: 40. https://www.iofbonehealth.org/ sites/default/files/media/PDFs/Fact Sheets/2014-Factsheet-Burden Osteoporosis-A4-ES.pdf

2. International Osteoporosis Foundation. Hip Fracture Incidence Map | International Osteoporosis Foundation. Available in: https://www. iofbonehealth.org/facts-and-statistics/hip-fracture-incidence-map

3. Maggi S, Kelsey JL, Litvak J, Heyse SP. Incidence of hip fractures in the elderly: a cross-national analysis. Osteoporos Int. 1991; 1(4): 232-41.

4. Azagra R, Martin-Sánchez JC, Aguyé A. Europe PMC Funders Group Changing trends in the epidemiology of hip fracture in Spain. Osteoporos Int. 2014; 25(4): 1267-74. doi: 10.1007/s00198-013-2586-0.

5. Cooper C, Campion G, Melton LJ. Hip fractures in the elderly: a world-wide projection. Osteoporos Int. 1992; 2(6): 285-9. Available in: http://www.ncbi.nlm.nih.gov/pubmed/1421796

6. Mow TC, Lukeis J, Sutherland AG. The benefits of streamlined hip fracture management in a regional hospital. Geriatr Orthop Surg Rehabil. 2017; 8(2): 99-103. doi: 10.1177/2151458516689284.

7. Clark P, Lavielle P, Franco-Marina F, et al. Incidence rates and life-time risk of hip fractures in Mexicans over 50 years of age: a population-based study. Osteoporos Int. 2005; 16(12): 2025-30. doi: 10.1007/s00198-005-1991-4.

8. Alarcón T, Gonzalez-Montalvo JI, Gotor P, Madero R, Otero A. A new hierarchical classification for prognosis of hip fracture after 2 years' follow-up. J Nutr Health Aging. 2011; 15(10): 919-23. doi: 10.1007/ s12603-011-0129-y.

9. Ojeda-Thies C, Sáez-López P, Currie CT, et al. Spanish National Hip Fracture Registry (RNFC): analysis of its first annual report and international comparison with other established registries. Osteoporos Int. 2019; 30(6): 1243-54. doi: 10.1007/s00198-019-04939-2.

10. Hashmi A, Ibrahim-Zada I, Rhee P, et al. Predictors of mortality in geriatric trauma patients. J Trauma Acute Care Surg. 2014; 76(3): 894-901. doi: 10.1097/TA.0b013e3182ab0763.

11. Sáez P, González-Montalvo JI, Alarcón-Alarcón T, Gotor-Pérez P, Martín-Maestre I. ¿Es posible mejorar la atención al paciente con fractura de cadera? Aportaciones del programa Fonda. $3^{a}$ Actual en Ortogeriatría Ávila. 2015; 308.

12. Viveros-García JC, Torres-Gutiérrez JL, Alarcón-Alarcón T, Condorhuamán-Alvarado PY, Sánchez-Rábago CJ, Gil-Garay E G-MJ. Fractura de cadera por fragilidad en México: ¿En dónde estamos hoy? ¿Hacia dónde queremos ir? Acta Ortop Mex. 2018; 32(6): 334-41.

13. Alsawadi A, Loeffler M. Graduated compression stockings in hip fractures. Ann R Coll Surg Engl. 2012; 94 (7): 463-7. doi: 10.1308/00 $3588412 X 13171221592492$.

14. National Institute of Health and Care Excellence (NICE). Hip fracture management (CG124). 2011; 1-19. Available in: https://www. nice.org.uk/guidance/cg124/resources/hip-fracture-managementpdf-35109449902789.

15. Approvals R, Ethics F. Best practice tariff (BPT) for fragility hip fracture care user guide. 2010; 1-10. Available in: http://www.dh.gov. uk/pbr\%0Ahttp://www.nigb.nhs.uk/ecc\%0Ahttp://www.nigb.nhs.uk/ ecc/meetings/

16. Prestmo A, Hagen G, Sletvold O, et al. Comprehensive geriatric care for patients with hip fractures: A prospective, randomised, controlled trial. Lancet. 2015; 385(9978): 1623-33. doi: 10.1016/S01406736(14)62409-0.

17. González MJI, Gotor PP, Martín VA, et al. La unidad de ortogeriatría de agudos. Evaluación de su efecto en el curso clínico de los pacientes con fractura de cadera y estimación de su impacto económico. Rev Esp Geriatr Gerontol. 2011; 46(4): 193-9. doi: 10.1016/j. regg.2011.02.004.

18. Neuburger J, Currie C, Wakeman R, et al. Increased orthogeriatrician involvement in hip fracture care and its impact on mortality in England. Age Ageing. 2017; 46(2): 187-92. doi: 10.1093/ageing/ afw201.

19. González-Montalvo JI, Teresa A, Gotor P, Río M Del, Sáez P, Bárcena A. La intervención geriátrica puede mejorar el curso clínico de los ancianos frágiles con fractura de cadera. [Geriatr Manag frail Elder with hip Fract may Improv their clinical outcome]. Med Clin (Barc). 2001; 116: 1-5. doi: 10.1016/S0025-7753(01)71699-5.

20. Giusti A, Barone A. Optimal setting and care organization in the management of older adults with hip fracture. Eur J Phys Rehabil. 2011; 47(2): 281-96. doi: 10.4081/gc.2015.5602. 
21. Buecking B, Timmesfeld N, Riem S, et al. Early orthogeriatric treatment of trauma in the elderly: a systematic review and metaanalysis. Dtsch Arztebl Int. 2013; 110(15): 255-62. doi: 10.3238/ arztebl.2013.0255.

22. Pareja Sierra T, Rodríguez Solis J, Alonso Fernández P, Torralba González de Suso M, Hornillos Calvo M. Intervención geriátrica en el anciano ingresado por fractura de cadera en el Hospital Universitario de Guadalajara: repercusión clínica, asistencial y económica. Rev Esp Geriatr Gerontol. 2017; 52(1): 27-30. doi: 10.1016/j. regg.2016.02.001.

23. Marsh D, Palm H. Rising to the challenge of fragility fractures. Injury. 2018; 49(8): 1392. doi: 10.1016/j.injury.2018.06.029.

24. Pioli G, Bendini C, Pignedoli P, Giusti A, Marsh D. Orthogeriatric comanagement-managing frailty as well as fragility. Injury. 2018; 49(8): 1398-402. doi: 10.1016/j.injury.2018.04.014.

25. Sabharwal S, Wilson H. Orthogeriatrics in the management of frail older patients with a fragility fracture. Osteoporos Int. 2015; 26(10): 2387-99. doi: 10.1007/s00198-015-3166-2.

26. Falasci P, Marsh D. Orthogeriatrics. (Falaschi P, Marsh DR, eds.). Cham: Springer International Publishing; 2017. doi: 10.1007/978-3319-43249-6.

27. Viveros-García J. Ortogeriatría en México: un futuro posible. Med Int Méx. 2019; 35(1): 150-3. doi: 10.24245/mim.

28. Mehrholz J, Wagner K, Rutte K, Meissner D, Pohl M. Predictive validity and responsiveness of the functional ambulation category in hemiparetic patients after stroke. Arch Phys Med Rehabil. 2007; 88(10): 1314-19. doi: 10.1016/j.apmr.2007.06.764.

29. Duarte-Flores JO. Las unidades de ortogeriatría en México son una prioridad. Rev Med Inst Mex Seguro Soc. 2018; 56(4): 332-33.

30. Dinamarca MJ, Améstica LG, Carrasco BA. Mortalidad intrahospitalaria en adultos mayores chilenos con fractura de cadera: incidencia, causas y otros elementos de interés. Rev Chil Ortop y Traumatol. 2018; 59(02): 041-6. doi: 10.1055/s-0038-1668593.

31. Dinamarca-Montecinos JL, Améstica-Lazcano G, Rubio-Herrera R, Carrasco-Buvinic A, Vásquez A. Características epidemiológicas y clínicas de las fracturas de cadera en adultos mayores en un hospital público chileno. Rev Med Chil. 2015; 143(12): 1552-9. doi: 10.4067/ S0034-98872015001200008.
32. Ortogeriatría. 2016. Available in: https://www.fsfb.org.co/wps/portal/fsfb/ inicio/servicioensalud/servicios-y-especialidades/sectionItem/ortogeriatria

33. Sáez-López P, Ojeda-Thies C, Alarcón T, et al. Registro Nacional de Fractura de Cadera.; 2018. Available in: http://rnfc.es/wp-content/ uploads/2019/11/Informe-Anual-RNFC-2018.pdf

34. Royal College of Physicians. National Hip Fracture Database (NHFD) Annual Report 2019.; 2019. Available in: https://www.nhfd.co.uk/

35. ANZHFR. ANZHFR Bi-National Annual Report for Hip Fracture Care 2017.; 2017.

36. Scottish Hip Fracture Audit. Scottish Hip Fracture Care Pathway Report 2017. 2017: 1-44. Available in: www.shfa.scot.nhs.uk

37. Gjertsen JE, Engesaeter LB, Furnes O, et al. The norwegian hip fracture register: experiences after the first 2 years and 15,576 reported operations. Acta Orthop. 2008; 79(5): 583-93. doi: 10.1080/17453670810016588.

38. Cañon AP. Correlación del estado funcional y nutricional en ancianos hospitalizados con fractura de cadera en un hospital de alta complejidad. Univ Méd ISSN 0041-9095. 2015; 56(2): 136-44. doi: 10.1109/ciced.2018.8592188.

39. CB JL. Artroplastía de cadera secundaria a fractura en el hospital de atención integral del adulto mayor en Quito. 2019; 4(1). Tesis. doi: 1037//0033-2909.I26.1.78.

40. Baroni M, Serra R, Boccardi V, et al. The orthogeriatric comanagement improves clinical outcomes of hip fracture in older adults. Osteoporos Int. 2019; 30(4): 907-16. doi: 10.1007/s00198-019-04858-2.

41. Mitchell P, Akesson K. How to prevent the next fracture. Injury. 2018; 49(8): 1424-9. doi: 10.1016/j.injury.2018.06.031.

42. Akesson K, Marsh D, Mitchell PJ, et al. Capture the fracture: a best practice framework and global campaign to break the fragility fracture cycle. Osteoporos Int. 2013; 24(8): 2135-52. doi: 10.1007/s00198-013-2348-z.

43. Marcantonio ER, Flacker JM, Wright RJ, Resnick NM. Reducing delirium after hip fracture: a randomized trial. J Am Geriatr Soc. 2001; 49(5): 516-22. Available in: http://www.ncbi.nlm.nih.gov/ pubmed/11380742

44. Chuan A, Zhao L, Tillekeratne N, et al. The effect of a multidisciplinary care bundle on the incidence of delirium after hip fracture surgery: a quality improvement study. Anaesthesia. 2020; 75(1): 63-71. doi: 10.1111/anae.14840. 